\title{
Framework of Gesture for blind people usable at touch mobile phones
}

\author{
Shabnam Mohamed Aslam \\ Assistant Professor Department of Computer Applications SRM University \\ Govindarajan Swaminathan \\ Professor Department of Computer Applications SRM University sgrsgr.yahoo.com
}

\begin{abstract}
The world's population is comprised of, ot only people who can see, but also those who cannot see. Rarely, we find devices and technological advances that promote the accessibility for the visually impaired. Definitely, fully touch enabled devices are available today, but their primary focus is not making it accessible for the blind people by use of advanced GPS technology, Data Connectivity, Gesture Recognition and Image Recognition. This paper presents the the overall architectural design mobile operating system named Eyedroid and the framework of gesture interaction system for differently-abled people in a mobile device. Gesture input method prototype developed and tested with blind users.Basic usability factors such as easiness of handling, comfort ability easiness of entering text, words per minute were analyzed to measure gestural system performance.
\end{abstract}

Keywords- Android, accessibility, blind, eyes-free, impaired, interaction system, mobile interface, mobile interface design, differently abled, Eyedroid, eyes-free.

\section{Introduction}

In this paper section-II to section-VI presents the need for new mobile technology that can help in making it easier for the blind people to access things. It focuses on development of a fully fledged Operation System based on Android specially designed only for the visually impaired. Section-IX describes the layered architectural design of mobile operating system named Eyedroid and section $\mathrm{X}$ and section XI shows the Braille based gesture framework for entering a text and pilot test reports respectively.

\section{Need for the Study}

In rarest of the cases, it is observed that modern technological advances are made for the accessibility and usability of visually impaired. Such technology is either tough to implement or very costly. Most of the Mobile Phone companies provide phones with overwhelming user interfaces, features that may or may not be used by everyone who uses the phone. But these high end features and user interface are totally useless for people who cannot see. So the need of this study gets simplified to building a touch enabled mobile phone operating system that doesn't have any clumsy features that make it is difficult to use and without any interface. With MultiTap most errors occurred due to difficulties in multitapping, more specifically in finding the right timing to navigate between characters of a group. This was particularly apparent in the beginning, as some users would tend to not time well their taps, resulting in accepting undesired characters. Even though most are perfectly accustomed to multi-tap on their mobile keypads, some users had difficulty adapting this technique to a sensitive touch device. These adaptation difficulties were also apparent with the NavTouch method. Users would frequently touch/rest their fingers on the screen resulting in errors. Some users would also accidently fail doing the directional gestures, tapping the screen instead of actually doing fling gestures. A concern of some users was the difficulty they found in keeping track of the current text, as they would tend to get confused or even forget the current state of the text as they navigated through the alphabet

\begin{abstract}
Objectives
Taking into consideration the needs of this study, the focus of research has been restricted to find methods by which a person with visual disability and dump can "Interact" with a highly advanced operating system that can help them know each and everything in and around them. The word differently enabled in our title refers to the person who is physically challenged. This paper gives the overall idea of developing the system, and as a first step the interaction method is developed and presented in this paper.
\end{abstract}

\section{Hypothesis}

Throughout the study, the focus has been made to find each and every possibility to allow blindfold interaction with the Android phone. To achieve my stated objective, the possible hypothesis is to make use of modern Gesture Recognition technology [2] to help interaction with the mobile phone screen, usage of Camera for image recognition and GPS for the location based implementation of features.

\section{Scope of Research}

This custom built Android Operating System provides properly implemented mechanism and standardized ways to interact with the phone. It bridges the gap between vision loss and vision power. Blind people do not need to worry about an array of factors that highly affect their day to day life and thus make it difficult to live their life in a simple manner.

a) The primary situation where a blind person can interact with the system is through gestures. All gestures would be planned as per universally known standards of Braille based typing [4]. Basic gestures means, swiping with the finger on any part of the screen or tapping with one or multiple fingers. This 
would be the key point through which all other features of this system would be undertaken for development.

b) Take into consideration a situation where a person with vision disability would urgently need to communicate with his/her guardian. The system would be providing a mechanism that would automatically send the current location of the former to the latter.

c) Another scenario, where the blind person wants to send an SMS. The Operating System would provide an easy gesture based typing facility through which the SMS can be typed and send to the receiver

d) Consider one situation where the blind person received a new message. Here the system would instantly notify the person about the incoming message and would read out the message with use of advanced customized text-to-speech facility.

e) In a situation where, the person wants to make a call, there would be a redefined, customizable and manipulation capable functionality in the operating system that would help to make calls based on the same gesture based typing.

f) The visually impaired person would also be able to recognize various real world objects by means of Advanced Image Recognition and Augmented Reality. Currently the focus of the study will be limited to identify currency notes and various other simple objects. The system would capture snaps of the object through the camera and identify the currency note, and give a voice based feedback to the user of what the object or note is.

g) The system would be having advanced GPS enabled path tracking mechanisms that would guide the person on each place and help him walk or travel easily. The system would make use of GPS technology to track the current location of the concerned person on the map and guide the person to his/her destination.

h) Consider a situation where the person wants to use email facility. In such situations, the system would enable to write emails to the sender using gesture based typing and read out incoming emails.

i) The system would also focus on patterned vibration feedback [1] on each moves and gestures to confirm that the action was properly done.

j) The user would be getting continuous voice based support from the mobile phone system for each and every activity performed along with the gesture's vibration feedback.

k) These are basic functionalities and small visualization of the actual ideology for the development of this mobile phone system. Future developments and advances would be focusing on more sophisticated error free system that would completely fill the gap between vision enabled mobile phone usage and the opposite.

\section{Socio - Economic Benefits}

Modern cell phone hardware are capable of computing at really high speeds, available at really cheap prices and tremendously customizable. Development of a truly dedicated mobile phone operating system would prove to be beneficial on both ways.

a) Social benefits include the invasion of modern Android based cell phones into a completely untouched section of the society. People without vision, would be able to make use of modern mobile computing power and features. Interact with the smart operating system and benefit from it.

b) From the economic point of view it can be seen, that instead of fully developing a new operating system for the blind, from scratch. It would be highly cost effective to develop a custom built Android system. Android Operating System is open source in nature thus it can drastically decrease the cost of development of the system for the current study.

c) EyeDroid promise many benefits to mobile communications and people who are travelling on long journeys. It could help a travelling individual to be in continuous guidance of the operating system and advanced technologies.

\section{Review of Related Literature}

With the advent of sixth sense technology "gesture computing" and the mobile technology makes the blind people to have better mobile accessibility. Mobile Usability by blind people and favorite gestures of blind people are derived [1] as swipe the screen left to right, right to left, top to down, From down to top to access different options of mobile. Users use the touch screen[2] in different ways such as swipe left to right, Using Qwerty keyboard on touch screen, multi touch method and Braille typing. But according to our best knowledge touch screen supports Braille reading only. But in our work we have introduced Braille inputing (writing) method. This [3] paper reveals the common mobile tasks being used by blind person and the frequency of mobile application accessibility. They have designed four touch screen gestures to access mobile applications. [4] This research expose temporal tactile feedback and Braille coding can be used to transmit single-character information.

\section{Research Questions}

What is the nature of implementation of the Android based Operating System?, What would be the learning curve of the system after development?, Is the system extensible to support multiple languages?, Would it support OTA updates?

\section{Architectural Model}

This section describes the logical representation of the architectural model of the operating system that overlays the core Linux based Android System and Kernel. Basically, the architecture of this system would be to completely modify the top most layer of an Android Operating System and lay new libraries and a complete framework for the functionalities 
needed for a blind person. Many of the core libraries would be taken use of. Here, the bottom most layer, Linux kernel is the core of any Android system. The second layer is a set of system libraries and some middle ware that help in functioning the hardware buttons and components. Above it is the actual core implementation of the Android development suite that helps us to implement basic functionalities such as touch detection, GPS libraries, RIL libraries etc. And the topmost layer would actually be the implementation of all core EyeDroid libraries and application framework.

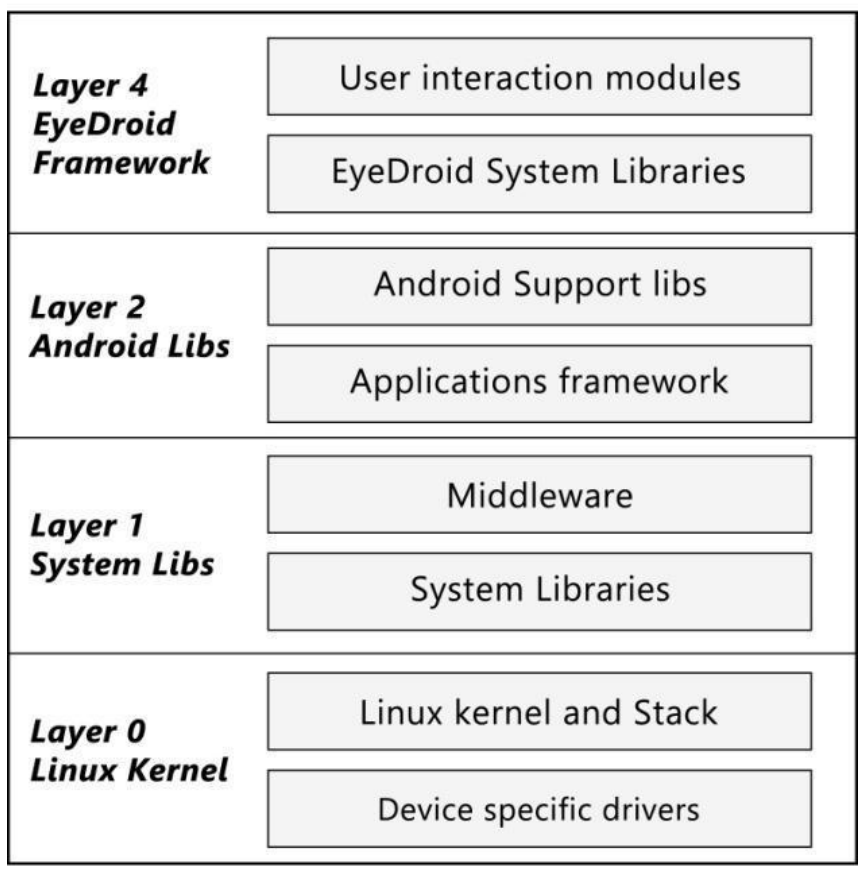

Fig.1.Eydroid system

\section{A.Technologies to build the system}

Based on the architectural design of the system, the Android SDK and NDK would be required as a primary programming environment.

\section{B.Hardware requirements for system}

Hardware specification of the system are mentioned in details in table no. 1 .

\section{TABLE 1. Hardware Specifications}

\begin{tabular}{|l|l|}
\hline Hardware Details & Minimum Reqd. \\
\hline Screen size & $>3.2$ inches \\
\hline Multi touch & Needed for gestures \\
\hline Resolution & Minimum $320 * 480$ \\
\hline Speakers & For audio feedback \\
\hline Vibration device & For haptic feedback \\
\hline Accelerometer & For motion sensing \\
\hline GPS & For location services \\
\hline Camera & At least 5mp \\
\hline Hardware buttons & To control volumes \\
\hline Motion sensor & To get shake gestures \\
\hline
\end{tabular}

\section{C.Design of User Interaction Module}

The users can interact with the mobile through gesture patterns developed based on Bralle key method. This system helps kind of users such as visually impaired, vocally challenged and dum. The usage of haptic feedback enables deaf people also uses mobile phone. At first the basic functionalities considered are typing text, getting the feedback from the mobile. We introduce the new gesture based Braille text typing. The braille keypad consists of six dots for each character. The combination of embossed dot and disabled dot creates the pattern of one character. Five Gesture patterns are considered, based on which combinations of gestures for twenty six alphabets are created. The user gives three gesture to input one alphabet. The mobile device gives the audio feedback of entered character.

\section{i)Four Basic gestures \\ - Swipe left to right \\ - $\quad$ Swipe right to left \\ - $\quad$ Swipe Top to Bottom \\ - Swipe Bottom to Top \\ - Tap}

The mobile screen is divided into two columns and three rows that creates six cells. Each cell represent a Braille dot.

\section{Gesture patterns Input A

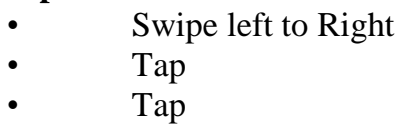 \\ Input B

$\begin{array}{ll}\text { - } & \text { Swipe left to right } \\ \text { - } & \text { Swipe left to right } \\ & \text { Tap }\end{array}$

Input $\mathbf{C}$

$\begin{array}{ll}- & \text { Bottom to top } \\ \text { - } & \text { Tap } \\ & \text { Tap }\end{array}$

Input D

- $\quad$ Bottom to top

- $\quad$ Right to left

- Tap

Twenty Six gesture patterns developed, and the Audio feedback received by the user. When the user follow the Input A method, A will be typed, and the user gets the audio feedback as " you typed A". The Interface design is completed, that is the top layer is designed in the architecture framework. The gesture input prototype has been designed using Android 4.1 and implemented with an Android mobile phone. 


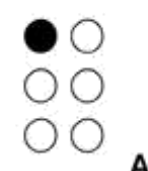

A
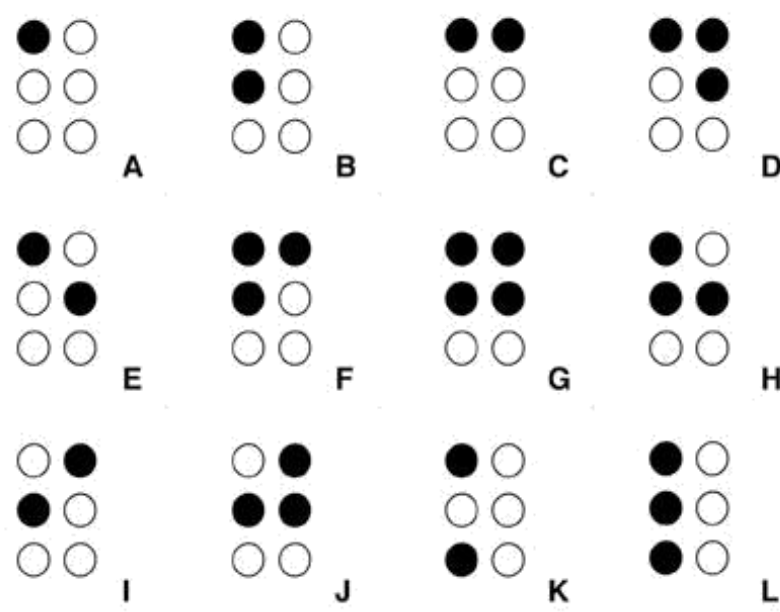

L

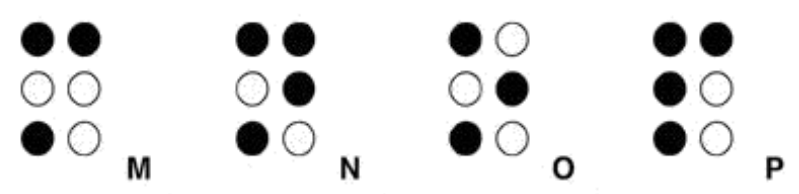

$\because$
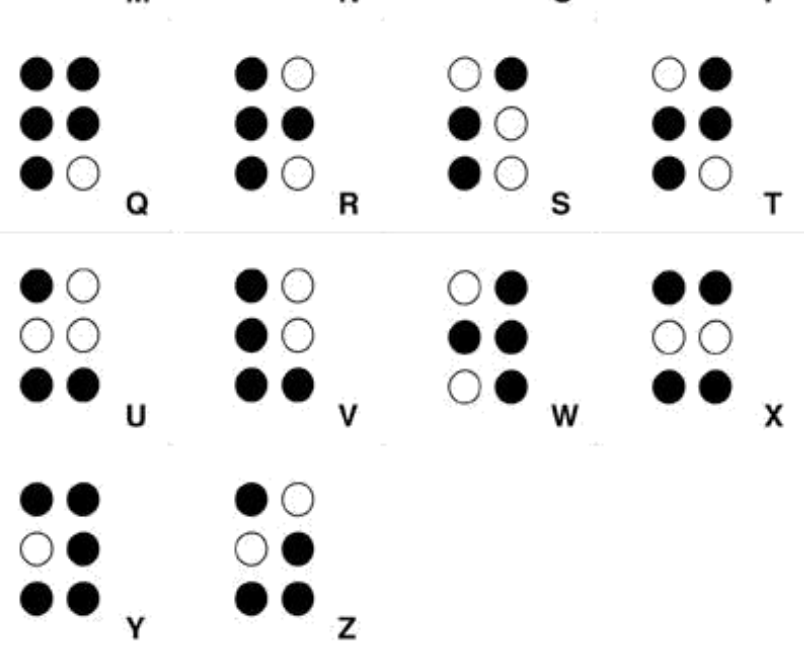

Fig.2-Braille Key

\section{ii) Pilot Test}

The gesture input prototype of gesture input method has been developed and tested with five fully blind people of age group from 20 to 60, Braille known; and the necessary training and feedback collected on some of the mobile usability measures such as
a) Easiness of entering text
b) Adaptability
c) Comfort ability
d) Easiness of handling
e) Occurrence of Error
f) Rate of interest to use the system
g) Words per minute

Table 1 depicts the score given by five subjects for various usability factors of Eyedroid method. The factors marked with * are give high score rate. During the test the subjects felt happy about no need to trace the screen for selecting a particular character; rather they give the gesture freely anywhere in the screen for a particular Braille code for a particular character.

Subjects have more likeliness towards 'Easiness of entering the text', 'Comfort ability' (as they use single hand to hold the phone as well as to give gesture), 'Rate of interest to use the system' (as growing phase of touch interaction in the market, they are eager to learn and adapt to gesture base interaction system), 'Words per minute' (as arrive to maximum rate as compared with existing methods).Subjects makes lots of error when starting to use the system but the error rate gradually reduced after little practice. Types of errors committed

$>\quad$ Miss-gesture

$>\quad$ wrong gesture-Braille mapping

$>\quad$ finger miss-positioning on the screen

\section{TABLE.2.Pilot test result}

\begin{tabular}{|l|c|c|c|c|c|}
\hline Usability factors & P1 & P2 & P3 & P4 & P5 \\
\hline Easiness of entering text* & 9 & 8 & 10 & 9 & 8 \\
\hline Adaptability & 5 & 4 & 8 & 5 & 6 \\
\hline Comfort ability* & 9 & 8 & 9 & 8 & 9 \\
\hline Easiness of handling & 5 & 6 & 5 & 6 & 6 \\
\hline Occurrence of Error & 5 & 4 & 8 & 6 & 7 \\
\hline $\begin{array}{l}\text { Rate of interest to use the } \\
\text { system* }\end{array}$ & 9 & 10 & 9 & 10 & 9 \\
\hline Words per minute* & 11 & 12.6 & 13 & 11 & 11 \\
\hline
\end{tabular}

Double tap gesture was used for backspace, the characters corrected by back space or clear option. Clear option is performed by long tap on the screen. When the subject holds the mobile on left hand and gesture with index finger of right hand reduces miss-gesture type of error and the learning of gesture-Braille mapping reduces the mapping type of error. Long term training and Eyedroid customization with blind people will reduce the error rate drastically. The feedback given by the subjects ensures the easiness of use of Eyedroid motivates ourselves to do research with the aim of devising a full-fledged dedicated touch handheld system for physically challenged people. The prototype screen has no layouts, the user is free to gesture at any position over the entire screen area institutes avoidance of spatial problem.

\section{Socio Economic Recommendations}

This section discusses the socio economic requirement for the successful development and implementation of the system. Acceptance from the end users, viz. The actual blind people who face day to day problems in doing the simplest of the daily tasks. 


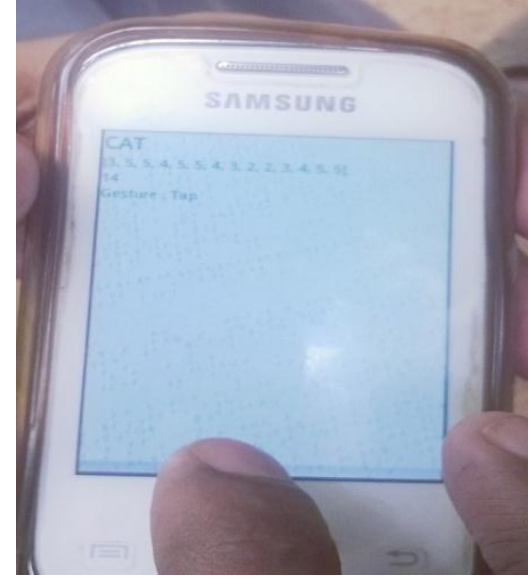

Fig 3 Gesture input Prototype screen

Such a system would be useful only if it is actually being used by the visually impaired people and accepted as a daily use device. The system would be having all the necessities that are needed to communicate, travel, respond to events and various other things that modern mobile phones are capable of doing.

\section{Conclusion AND Future work}

In this paper the architectural design has been designed and the basic functionalities to be provided in the system have been stated. In future, the system would be inculcating various other sections of development, including the basic mobile activities such as messaging, calling mailing, hearing music and advanced image recognition for object recognition. The system would also provide human face recognition facilities. Further improvement would be made to improve localization of the language support.

\section{References}

1. Shaun K. Kane, Jacob O. Wobbrock, Usable gestures for blind people: Understanding preference and performance, Richard E. Ladner, ACM Transactions 2011.

2. João Oliveira Tiago Guerreiro Hugo Nicolau Joaquim Jorge Daniel Gonçalves Blind People and Mobile Touch-based Text-Entry: Acknowledging the Need for Different Flavors, ASSETS'11, October 2426, 2011,ACM

3. Shaun K. Kane,1 Jeffrey P. Bigham2 and Jacob O. Wobbrock, Slide Rule: Making Mobile Touch Screens Accessible toBlind People Using MultiTouch Interaction Techniques, ASSETS'08, October 13-15, 2008, ACM

4. 1Rantala, J. ; Dept. of Comput. Sci., Univ. of Tampere, Tampere ; Raisamo, R. ; Lylykangas, J. ; Surakka, Methods for Presenting Braille Characters on a Mobile Device with a Touchscreen and Tactile Feedback, Haptics IEEE Transactions, volume 2, Issue 1
5. Calle Sjöström, Using haptics in computer interfaces for blind people, CHI EA '01 CHI '01 Extended Abstracts on Human Factors in Computing Systems, Pages 245-24, ACM Digital Library

6. Felt, Adrienne Porte; Chin, Erika; Hanna, Steve; Song, Dawn; Wagner, David, CCS '11 Proceedings of the 18th ACM conference on Computer and communications security, Pages 627-638, ACM Digital Library

7. Jakob Nielsen, Gestural interfaces: a step backward in usability, Volume 17 Issue 5, September + October 2010, Pages 46-49, ACM Digital Library.

8. Stanford Course yields Braille based touch typing, //http://www.the-digital-reader.com/2011/10/18/newapp-turns-a-tablet-into-a-braillekeyboard/\#.UTATWDnRHIU

9. Kane, S., et al. 2011. Usable Gestures for Blind People: Understanding Preference and Performance. In Proc.of CHI

10. T.E. Starner and A. Pentland, "Visual Recognition of American Sign Language Using Hidden Markov Models,' Proc. Intl'1 Workshop Automatic Face and Gesture Recognition, IEEE Press, 1995, pp. 189-194.

11. 2. S. Lenman, L. Bretzner, and B. Thuresson, Computer Vision Based Hand Gesture Interfaces for Human-Computer Interaction, tech. report TritanaD0209, Centre for User Oriented IT Design, June2002.

12. Buxton, W., Foulds, R., Rosen, M., Scadden, L. and Shein, F. Human interface design and the handicapped user. SIGCHI Bulletin 17, 4 (1986), 291-297

13. American Association of the Deaf-Blind http://aadb.org

14. McGookin, D., Brewster, S., and Jiang, W. 2008. Investigating touchscreen accessibility for people with visual impairments. $5^{\text {th }}$ Nordic Conference on Human-Computer interaction: Building Bridges (NordiCHI $\square 08$ ).

15. Bonner, M. et al. 2010. No-Look Notes: Accessible Eyes-Free Multitouch Text-Entry. Pervasive Computers, 409-426.

16. Gregor, P. and Newell, A. 2001. Designing for dynamic diversity: making accessible interfaces for older people. In Proceedings of the 2001 EC/NSF work-shop on Universal accessibility of ubiquitous computing, 90-92

17. Yfantidis, G., and Evreinov, G. 2006. Adaptive Blind Interaction Technique for Touchscreens. UAIS, 4, 328-337 


\section{Biographical Notes}

Shabnam Aslam obtained her Bachelor's degree in Computer Science from Bharadhidasan University in 2001. Then she obtained her Master's degree in Computer Science and is pursuing $\mathrm{PhD}$ in Computer Science majoring in human computing interaction, and gesture computing from SRM University, India. Currently, she is an Assistant Professor at the Faculty of Science and Humanities, SRM University, India. Her specialisations include Gesture computing, HCI, cloud computing technologies. Her current research interests are analyzing the blind users' interaction with various types of mobile designs, research on Braille code and developing a gesture-based interaction methodology for visually impaired users, big data, cloud security management.

Swaminathan Govindarajan received his BSc degree from Madras University, MSc in Engineering from PSG college of Technology, Coimbatore, and PhD in Information Technology (IT) from Faculty of Engineering Madras University.Earlier, he had served major software MNC as General Manager. Currently, he works for SRM University as a Professor and Head for Computer Applications Department (PG) since 2009. He has published more than ten journals, which include five international publications. His research interests are HCI, ERP and software processes. 